\title{
Semi-automatic Segmentation of Skin Lesions based on Superpixels and Hybrid Texture Information ${ }^{\star}$
}

\author{
Elineide S. dos Santos ${ }^{\mathrm{a}}$, Rodrigo de M. S. Veras ${ }^{\mathrm{a}}$, Kelson R. T. Aires ${ }^{\mathrm{a}}$, Helano M. B. F. Portela ${ }^{\mathrm{a}}$, Geraldo Braz Junior ${ }^{\mathrm{b}}$, Justino D. \\ Santos $^{\mathrm{a}}$, João Manuel R. S.Tavares ${ }^{\mathrm{c}, *}$ \\ ${ }^{a}$ Department of Computing, Federal University of Piauí - Teresina, Brazil \\ ${ }^{b}$ Department of Informatics, Federal University of Maranhão - São Luís, Brazil \\ ${ }^{c}$ Instituto de Ciência e Inovação em Engenharia Mecânica e Engenharia Industrial, Departamento de Engenharia Mecânica, Faculdade de Engenharia, \\ Universidade do Porto, Porto, Portugal
}

\section{A R T I C L E I N F O}

\section{Article history:}

Received 1 May 2021

2000 MSC: 41A05, 41A10, 65D05, $65 \mathrm{D} 17$

Keywords: Texture information, computer-aided diagnosis, melanoma, dermatoscopic image segmentation.

\begin{abstract}
A B S T R A C T
Dermoscopic images are commonly used in the early diagnosis of skin lesions, and several computational systems have been proposed to analyze them. The segmentation of the lesions is a fundamental step in many of these systems. Therefore, a semi-automatic segmentation method is proposed here, which begins by building the superpixels of the image under analysis based on the zero parameter version of the simple linear iterative clustering (SLIC0) algorithm. Then, each superpixel is represented using a descriptor built by combining the grey-level co-occurrence matrix and Tamura texture features. Afterward, the gain ratios of the features are used to select the input for the semisupervised seeded fuzzy C-means clustering algorithm. Hence, from a few specialistselected superpixels, this clustering algorithm groups the built superpixels into lesion or background regions. Finally, the segmented image undergoes a post-processing step to eliminate sharp edges. The experiments were performed on 1,380 images: $401 \mathrm{im}$ ages from the $\mathrm{PH}^{2}$ and DermIS datasets, which were used to establish the parameters of the method, and 3,573 images from the ISIC 2016, ISIC 2017 and ISIC 2018 datasets were used for the analysis of the method's performance. The findings suggest that, by manually identifying just a few of the generated superpixels, the method can achieve an average segmentation accuracy of $96.78 \%$, which confirms its superiority to the ones in the literature.
\end{abstract}

(C) 2022 Elsevier B. V. All rights reserved.
${ }^{\star}$ Semi-automatic Segmentation of Skin Lesions.

*Corresponding author: Rua Dr Roberto Frias s/n, 4200-465 Porto, Portugal

e-mail: elineide.silva@ufpi.edu.br (Elineide S. dos Santos), rveras@ufpi.edu.br (Rodrigo de M. S. Veras), kelson@ufpi.edu.br (Kelson R. T. Aires), helano38@gmail . com (Helano M. B. F. Portela), geraldo.braz@ufma.br (Geraldo Braz Junior),

justinoduarte@gmail.com (Justino D. Santos), tavares@fe.up.pt (João Manuel R. S.Tavares)

\section{Introduction}

In the human body, one can find the integumentary system, usually known as skin, whose primary functions are excretory, protective, relationship, thermoregulatory and metabolic. However, in addition to other factors, when the skin is repeatedly exposed to the sun over long periods, there is a high possibility for occurring skin lesions (Lacy and Alwan, 2013).

Skin lesions can be benign or malignant. Benign lesions have more regular edges and uniform coloring (Figure $1(\mathrm{a})$, whereas malignant lesions have more irregular borders and varied color- 
ing (Figure 1(b)). Malignant lesions can lead to skin cancers, such as melanoma, which is a very aggressive cancer with easy proliferation. A recent report from The Skin Cancer Foundation (Organization, 2019) stated that more than two people died from skin cancer each hour in the United States. On the other hand, the American Cancer Society estimated 1,898,160 cancer cases diagnosed, the equivalent of 5200 new cases each day in 2021, with 101,280 of them involving melanoma (Siegel et al. 2021).

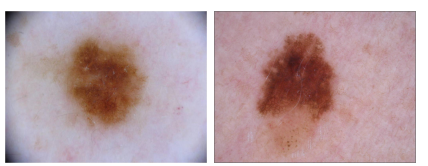

(a) benign lesions

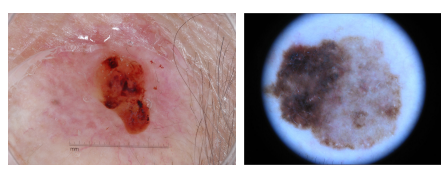

(b) malignant lesions
Fig. 1. Examples of skin lesions in dermoscopic images.

Skin cancer is often detected based on images that physicians visually analyze. However, manual image segmentation, i.e., the manual delineation of the lesion under analysis, is a highly time-consuming task which requires well skilled and trained physicists. In order to overcome these difficulties, ComputerAssisted Diagnosis (CAD) systems have been developed by combining techniques of artificial intelligence and digital image processing in order to assist physicists in their clinical routine (Scharcanski and Celebi, 2013).

Lesion segmentation is an essential step in a CAD system developed to detect and classify skin lesions. The image obtained from the segmentation step can provide features of the lesion under analysis that can be used to classify it as benign or malignant. According to Goyal et al. (2020), the segmentation of the lesion, i.e., the detection of its border, is vital to accurately locate it in the image under analysis and diagnose it. In addition, the Asymmetry, Border, Color and Diameter (ABCD) rule can help non-dermatologists to easily differentiate benign melanocytic naevi from melanoma (Abbasi et al. 2004). Recently, Moura et al. (2018, 2019) demonstrated the importance of the ABCD rule in the automatic diagnosis of melanoma from dermoscopic images.

In recent years, deep learning has gained considerable attention from the medical imaging analysis community (Sultana and Puhan, 2018, Chan et al., 2020). Usually, deep-learningbased systems also have a segmentation step, which is transparent to the user. Hence, in some of these systems, the lesion under analysis is segmented before being classified by a convolutional neural network (Xie et al., 2019).

Various methods have been proposed to process and analyze skin lesions in images (Khalid et al., 2016, Pennisi et al. 2016, Li and Shen, 2018, Filali and Belkadi, 2019). However, the researchers usually apply strategies that depend on the characteristics of the used image dataset, which is often private (López Sarachaga et al., 2019, Halder et al., 2020). Additionally, the segmentation becomes more difficult when there is a high diversity in the skin lesion images under analysis, such as when the images present different skin colors or were acquired under non-constant imaging conditions.
In this article, a semi-automatic method for the segmentation of skin lesions in dermoscopic images is proposed. The method was applied to a wide variety of skin lesion images collected from several publicly available datasets. Various experiments were performed using different texture descriptor algorithms and combined feature vectors in order to develop the proposed method. It includes a pre-processing step for hair detection and a pre-segmentation step based on superpixels. The user, e.g. the physician, then selects a few of the built superpixels, and the seeded fuzzy C-means (SFc-means) algorithm, proposed by Santos et al. (2018), is used to cluster the remainder ones as belonging to the skin lesion or to the image background. Finally, noise and fragments at the border of the segmented lesion are removed.

The remainder of this article is organized as follows. In Section 2, a survey of related works is presented. In Section 3, the proposed method is described. Section 4 provides details about the used image datasets and evaluation metrics, and presents the results. The findings are discussed in Section 5. The conclusions and recommendations for further studies are pointed out in Section 6 .

\section{Related Work}

Several methods have been proposed for the segmentation of skin lesions in images. According to the literature (Celebi et al. 2015; Oliveira et al., 2016; Ma and Tavares, 2016), the most common semi- and fully automatic segmentation methods can be classified into three groups: clustering, thresholding and deformable models. More recently, the use of deeplearning techniques has become popular (Sultana and Puhan. 2018). However, despite the numerous segmentation methods that have been developed, there is still a demand for improved performance (Chan et al. 2020).

In this study, an attempt was made to identify the methods that have been proposed to segment skin lesion images based on the used segmentation technique(s), obtained accuracy and publication year. Hence, a search was performed on three public databases: Scopus, IEEE Xplore and Web of Science, using three search strings: "skin lesion segmentation", "melanoma segmentation" and "dermoscopy image segmentation".

The following selection criteria were established:

- The articles must be published after 2015 and in the fields of computer science, engineering or medicine;

- Dermoscopy images must be used in the articles;

- Public image datasets must be used in the evaluation of the segmentation accuracy in the articles;

- Evaluation metrics suggested by the International Skin Imaging Collaboration (ISIC) must be used in the articles.

Regardless of the used segmentation technique(s), one could verify that most of the found skin lesion segmentation methods include pre- and post-processing steps.

Table 1 summarizes the studies found in the literature based on their year of publication, segmentation technique(s), number 
of validation images, number of image datasets and segmentation accuracy. In all the found studies, the images used for validation were gathered from the same dataset(s) used to adjust the method's parameters.

After a thorough literature study, it was realized that the performance of each method decreased when the images under analysis were more difficult to segment; mainly, because of uneven illumination, artifacts, noise and low contrast. To address these difficulties, a semi-automatic method, which requires only a quick and straightforward user's interaction, is proposed. Although the segmentation results depend on the user's interaction, the proposed method relieves the user of the challenging task of delineating the lesion. Consequently, the method is considerably less time-consuming, and enables the user to achieve segmentation results with high confidence since the user has an active and essential role in the segmentation process.

\section{Proposed Segmentation Method}

The proposed semi-automatic segmentation method uses seeds selected by the user that are related to the lesion and non lesion, i.e., image background, regions. The selected seeds are then inputted into a semi-supervised clustering algorithm. Then, post-processing is performed to exclude misclassified areas and remove noise from the segmented lesion border. Figure 2 depicts the proposed segmentation method.

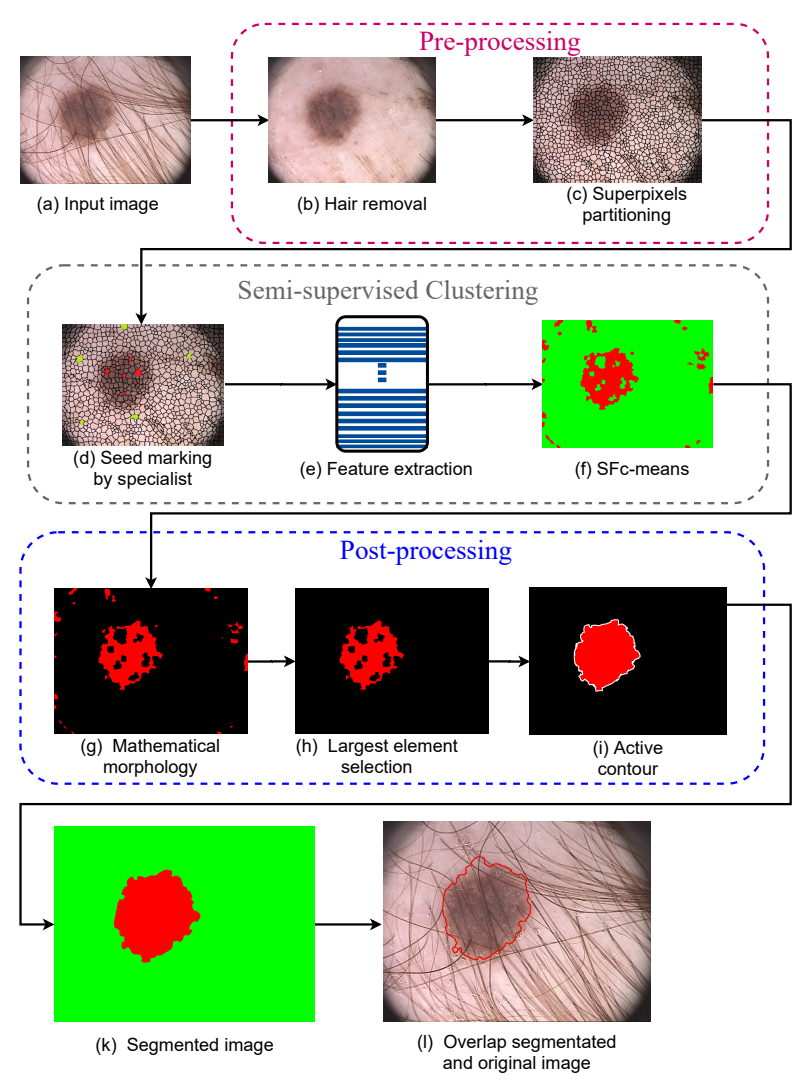

Fig. 2. Flowchart of the proposed segmentation method.

\subsection{Pre-processing}

\subsubsection{Hair removal}

The first step of the proposed method is devoted to hair removal. Hence, the DullRazor algorithm (Lee et al., 1997), which uses a morphological closing operation on grayscale images to identify regions with hair, is applied. Because hairs are long and thin structures, bilinear interpolation is then applied on the pixels of the identified hair regions. Finally, a median adaptive filter is used to smooth the modified hair pixels, as shown in Figure 3(b)

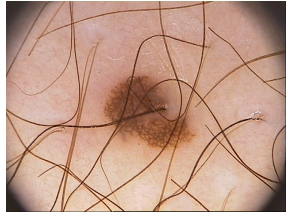

(a) Original image.

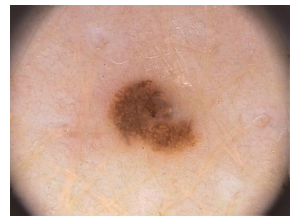

(b) Original image after the hair removal step.
Fig. 3. Example of hair removal in a dermoscopic image with a skin lesion.

\subsubsection{Superpixel partitioning}

Traditionally, in image processing, the pixels of an input image are individually processed; however, this requires high computational efforts when applied to images with large dimensions. In this study, superpixels are used to reduce this computational burden. Superpixels are typically generated by pixel clustering algorithms, which collect local redundancies in the input image and merge the corresponding pixels into superpixels. The proposed method uses a simple linear iterative clustering algorithm with zero parameters (SLIC0) (Nan and Mu. 2014), which, according to He and Chen (2017), has high computational efficiency for the generation of compact and uniform superpixels. The SLIC0 algorithm uses standard color and texture based features in the generation of the superpixels.

The SLIC0 algorithm selects the compactness parameter for each superpixel adaptively. The value of this compactness parameter is $\frac{c}{S}$, where $c$ is the number of superpixels, and $S$ is the area of the superpixel version. Hence, if $c$ is low, edges and border of the input image may be discarded, even if they have irregular shapes. The SLIC0 algorithm based on the Kmeans algorithm uses also information about color, brightness and spatial features. Thus, the superpixels are generated trough iterative clustering of similar pixels until they satisfy a convergence criterion.

To determine the optimal number of superpixels to be generated, different values were evaluated for $c$; mainly: 400, 600, $800,1000,1200,1400$ and 1600. Figure 4 shows the partitioning obtained according to different numbers of superpixels.

\subsection{Semi-supervised clustering}

The generated superpixels are then clustered using the SFcmeans algorithm, which groups input elements based on the knowledge obtained from labeled data; here, the superpixel seeds selected by the user. Notably, SFc-means is based on approximate reasoning (Ross, 2009) that involves fuzzy logic, 
Table 1. Summary of the studies found in the literature according to their publication year, segmentation technique(s), number of test images, number of test datasets, dataset(s) and segmentation performance(s).

\begin{tabular}{|c|c|c|c|c|}
\hline Work) & Technique(s) & N. of images & N. of datasets & Dataset, Performance \\
\hline Khalid et al. 2016 & Discrete Wavelet Transform & 200 & 1 & $\mathrm{PH}^{2}$, Accuracy: $93.87 \%$ \\
\hline Pennisi et al. 2016 & $\begin{array}{l}\text { Edge detection + Delaunay Triangula- } \\
\text { tion }\end{array}$ & 200 & 1 & $\mathrm{PH}^{2}$, Accuracy: $89.66 \%$ \\
\hline Li et al. 2018 & $\begin{array}{l}\text { 2D DenseUNet-167 architecture } \\
\text { Li et al. 2018. }\end{array}$ & 600 & 1 & ISIC 2017, Accuracy: $94.30 \%$ \\
\hline $\mathrm{Li}$ and Shen $\mathrm{Li}$ and Shen 2018 & Lesion Indexing Network & 150 & 1 & ISIC 2017, Accuracy: $95.00 \%$ \\
\hline Ünver and Ayan $\overline{2019}$ & \begin{tabular}{l|l|} 
GrabCut Algorithm & Rother et al. 2004$]$ \\
\end{tabular} & 800 & 2 & $\begin{array}{l}\mathrm{PH}^{2}, \quad \text { Accuracy: } \quad 92.99 \% \\
\text { ISIC } 2017 \text {, Accuracy: } 93.39 \%\end{array}$ \\
\hline Vasconcelos et al. 2019 & Morphological geodesic active contour & 200 & 1 & $\mathrm{PH}^{2}$, Accuracy: $94.59 \%$ \\
\hline Filali and Belkadi 2019 & Multi-scale contrast segmentation & 201 & 1 & DermIS, Accuracy: $97.86 \%$ \\
\hline Nida et al. $\mid 2019]$ & Deep regional CNN + Fuzzy C-means & 380 & 1 & ISIC 2016, Accuracy: $94.20 \%$ \\
\hline Fulgencio Navarro and Bescós [2019] & SLIC algorithm + Gaussian distribution & 600 & 1 & ISIC 2017, Accuracy: $95.50 \%$ \\
\hline Garcia-Arroyo and Garcia-Zapirain 2019 & $\begin{array}{l}\text { Cuts and their image masks + Histogram } \\
\text { thresholding + Mask post-processed }\end{array}$ & 979 & 2 & $\begin{array}{l}\text { ISIC 2016, Accuracy: } 93.40 \% \\
\text { ISIC 2017, Accuracy: } 88.40 \%\end{array}$ \\
\hline Goyal et al. 2020$\}$ & R-CNN with DeeplabV3C mask & 800 & 2 & $\begin{array}{l}\mathrm{PH}^{2}, \quad \text { Accuracy: } \quad 91.90 \% \\
\text { ISIC } 2017 \text {, Accuracy: } 94.08 \%\end{array}$ \\
\hline Xie et al. 2020 & Mutual bootstrapping deep CNNs & 800 & 2 & $\begin{array}{l}\mathrm{PH}^{2}, \quad \text { Accuracy: } \quad 96.50 \% \\
\text { ISIC } 2017 \text {, Accuracy: } 94.70 \%\end{array}$ \\
\hline Al-Masni et al. 2020 & Full resolution convolutional network & 2491 & 3 & $\begin{array}{l}\text { ISIC 2016, Accuracy: } 81.79 \% \\
\text { ISIC 2017, Accuracy: } 81.57 \% \\
\text { ISIC 2018, Accuracy: } 89.28 \%\end{array}$ \\
\hline Lei et al. 2020 & U-Net network + Dual discrimination & 1279 & 3 & $\begin{array}{l}\text { ISIC 2016, Accuracy: } 96.00 \% \\
\text { ISIC 2017, Accuracy: } 93.50 \% \\
\text { ISIC 2018, Accuracy: } 92.90 \%\end{array}$ \\
\hline 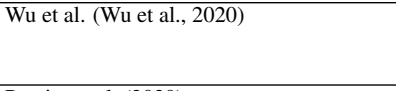 & $\begin{array}{l}\text { Dual encoder architecture }+ \text { Adaptive } \\
\text { dual attention module }+ \text { Multi-scale res- } \\
\text { olution }+ \text { Spatial information weighting }\end{array}$ & 1194 & 2 & $\begin{array}{l}\text { ISIC 2017, Accuracy: } 95.70 \% \\
\text { ISIC 2018, Accuracy: } 94.70 \%\end{array}$ \\
\hline Pereira et al. $[2020\}$ & $\begin{array}{l}\text { LBP processing + Space transformation } \\
+ \text { Clustering }\end{array}$ & 3500 & 3 & $\begin{array}{lcr}\text { Atlas, Jaccard: } & 84.50 \% \\
\text { PH }^{2} \text {, Jaccard: } & 86.30 \% \\
\text { Dermofit, Jaccard: } & 74.80 \% \\
\end{array}$ \\
\hline Hu et al. 2020 & $\begin{array}{l}\text { Enhanced fusion salience }+ \text { Adaptive } \\
\text { thresholding }\end{array}$ & 579 & 2 & $\begin{array}{l}\mathrm{PH}^{2}, \quad \text { Precision: } \\
\text { ISIC }\end{array}$ \\
\hline Arora et al. 2021, & $\begin{array}{l}\text { Modified U-Net network + Gaussian } \\
\text { blurring + Median filtering }\end{array}$ & 550 & 1 & ISIC 2018: 95.00\% \\
\hline Qamar et al. 2021 & $\begin{array}{l}\text { Combination of DenseNet network and } \\
\text { ResNet+ Atrous spatial pyramid pooling } \\
+ \text { dense skip connection }\end{array}$ & 518 & 1 & ISIC 2018: $96.25 \%$ \\
\hline Tong et al. (2021) & $\begin{array}{l}\text { Extended version of U-Net with triple at- } \\
\text { tention mechanism }\end{array}$ & 1179 & 3 & $\begin{array}{lcc}\mathrm{PH}^{2}, \quad \text { Accuracy: } & 92.60 \% \\
\text { ISIC } & \text { 2016, Accuracy: } & 95.40 \% \\
\text { ISIC } & \text { 2017, Accuracy: } & 94.30 \%\end{array}$ \\
\hline Araújo et al. 2021 & $\begin{array}{l}\text { Modified U-net network + Morphologi- } \\
\text { cal closing + Remove the artifacts + Fill } \\
\text { holes }\end{array}$ & 407 & 2 & $\begin{array}{l}\mathrm{PH}^{2}, \quad \text { Accuracy: } \quad 96.80 \% \\
\text { DermIS, Accuracy: } 98.30 \%\end{array}$ \\
\hline
\end{tabular}

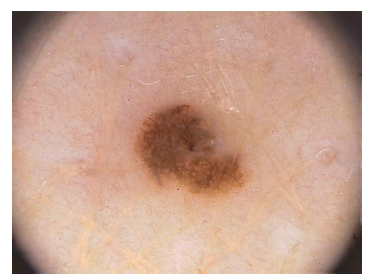

(a) Input image.

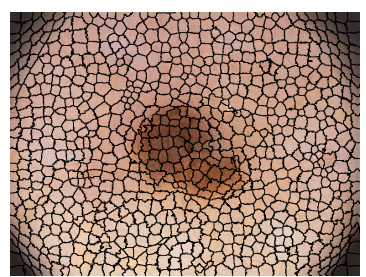

(c) $800 \mathrm{sp}$

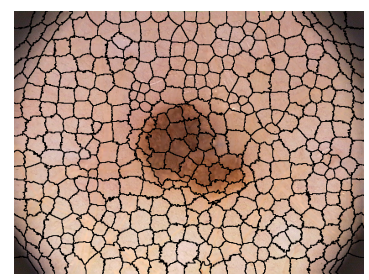

(b) $400 \mathrm{sp}$.

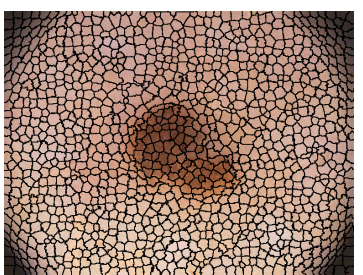

(d) $1200 \mathrm{sp}$.
Fig. 4. Examples of partitioning obtained according to different number of superpixels (sp.) (the black lines represent the border of each generated superpixel).

which enables the algorithm to take into account the imprecision inherent to the problem.

\subsubsection{Seeds selection}

The superpixels chosen by the user from the lesion and nonlesion regions are then inputted into the semi-supervised clustering algorithm. Figure 5 shows examples of such superpixels chosen by an user.

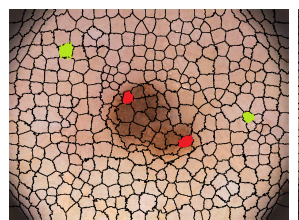

(a) Image with $400 \mathrm{sp}$

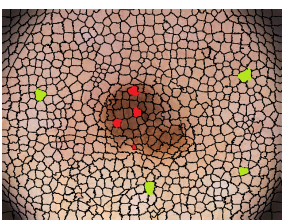

(b) Image with $800 \mathrm{sp}$

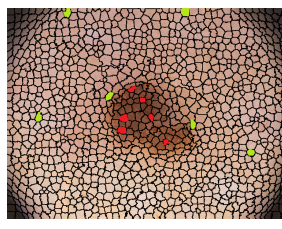

(c) Image with $1200 \mathrm{sp}$
Fig. 5. Images with user selected superpixels (sp.) (selected superpixels in red belong to the lesion region, and in green to the image background).

In this study, for each original image under analysis, the corresponding ground truth image, which is a binary image, was considered as its ideal segmentation. Figure 6 shows two examples of original images and their ground truths. As no physician was available to select the superpixel seeds to be use in the experiments, this user's interaction was simulated. Therefore, the seeds to be used by the SFc-means algorithm were randomly defined from the skin lesion and image background regions, 
which were identified based on the corresponding ground truth image. Also, in Santos et al. (2018), the authors used a variable cluster threshold and only $1 \%$ of the possible seeds to successfully segment different types of medical images. In our study, we performed initial tests using $1 \%, 2 \%$, and $5 \%$ of the superpixels as seeds; however, the results of the clustering process were similar. Therefore, we used only $1 \%$ of the superpixels as seeds since the lower is this number, less demanding is the required user interaction.

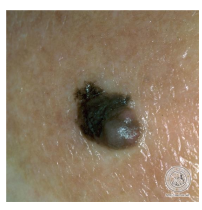

(a) Dermoscopic benign image.

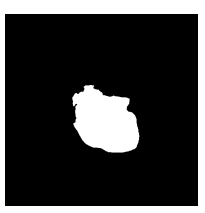

(b) Ground truth image of (a).

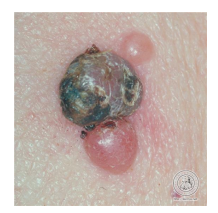

(c) Dermoscopic malignant image.

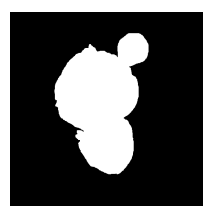

(d) Ground truth image of (b).
Fig. 6. Two original images and their corresponding ground truth segmented images.

\subsubsection{Texture feature extraction}

Features related to texture, color and shape can be extracted from input images, and their attributes can be grouped into feature vectors, known as "image descriptors". Here, the texture features are extracted from six color channels: the red, green and blue channels of the RGB space, and the lightness, greento-red variation and blue-to-yellow variation channels of the $\mathrm{L}^{*} \mathrm{a} \mathrm{b}^{*}$ space. Figure 7 presents an illustration of the used color channels of a skin lesion image. In the following sections, an overview of the used descriptors is given.

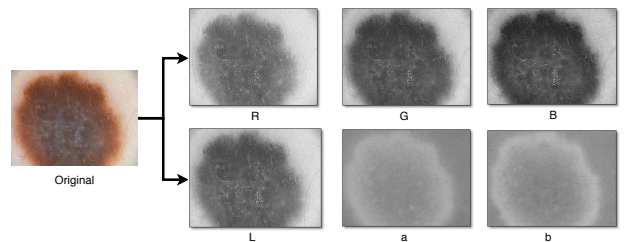

Fig. 7. Used color channels extracted from an original skin lesion image.

Grey-level co-occurrence matrix (GLCM): a GLCM (Haralick et al., 1973) is a texture descriptor used to analyze cooccurrences among pairs of image pixels in order to store the relative intensities in a square matrix. GLCM is used to compute the probability of co-occurrences between two gray levels, $k$ and $m$, according to an angle $\theta$, which was equal to $0,45,90$ and $135^{\circ}$ in the experiments presented here, and a distance that is known as "pixel pair spacing".

Several features can be defined from GLCM; however, the most commonly adopted are homogeneity, energy, correlation, contrast and entropy, which is invariant to rotation. Here, for each feature, seventeen attributes were extracted from each color channel, which led to 51 attributes extracted from the RGB space and 51 from the $\mathrm{L}^{*} \mathrm{a} * \mathrm{~b} *$ space for each feature.

Grey-level run-length matrix (GLRLM): this descriptor uses auxiliary matrices to check the repetition of computed pixels known as "primitives". In this study, for each cut in the image under analysis, a GLRLM was built that contained the number of grey-level primitives based on an angle $\theta$, which was equal to $0,45,90$ and $135^{\circ}$, and at a distance $d$. Hence, from this descriptor, 44 features were computed. Finally, the sum of all the GLRLM matrices was calculated and normalized by the number of replicates.

According to Galloway (1975), GLRLM provides five statistical measures: short-run emphasis, long-run emphasis, greylevel distribution, run-length distribution and run percentage. Chu et al. (1990) determined two further statistical measures: low grey-level run emphasis and high grey-level run emphasis.

Based on the idea of statistical measures, Dasarathyand and Holder (1991) described four additional feature extraction functions: short-run low gray-level emphasis, short-run high graylevel emphasis, long-run low gray-level emphasis and long-run high gray-level emphasis.

Geostatistics: it is a branch of statistics that is focused on populations with known addresses, i.e., coordinates. The fundamental theory of geostatistics is based on the assumption that, on average, samples near each other in terms of time and space are more similar than those that are distant (Isaaks and Srivastava, 1989).

In images, geostatistical functions are computed based on the values of pairs of pixels that correspond to a set of oriented vectors (distance and angle). Because the studied images have different dimensions, the same four angles used with the GLCM descriptor, i.e., $0,45,90$ and $135^{\circ}$, were used, and six distances, of which three were fixed (equal to 1,2 and 3 pixels) and the other three were proportional to the image width (equal to 40, 50 and $60 \%$ of the image width), were used. Hence, 24 oriented vectors were built.

In this study, four geostatistical functions were employed: semimadogram, semivariogram, correlogram and covariogram. These features represent the strength of the associations between responses as a function of distance and direction (Silva et al. 2004). Hence, the features describe the texture of a given image based on the degree of spatial association among its spatially referenced pixels (Sousa et al., 2017).

Local binary pattern (LBP): a thresholding operation is used to label each pixel of an input grayscale image (Ojala et al. 1996) into a binary pattern. This descriptor labels the pixels by examining the center of the $3 \times 3$ neighborhood window. An attribute vector containing 256 attributes is then built by fusing the collected values, and a histogram of the cells is built based on the actual numbers of zeros and ones.

Tamura: this descriptor, proposed by Tamura et al. (1978), contains nine attributes for each evaluated color channel and is associated with features that can be extracted from an image based on the human visual perception such as coarseness, contrast and directionality. Coarseness is a primary property, and, to some extent, it gives rise to texture, because it traduces the size of primitives in images. Contrast indicates the degree of clarity between different textured areas in an image. A high contrast region is the one that is more noticeable than the others. Direction is a global property of an image area, while orientation is related to the form and placement rules of primitives. Given a scenario where two primitives are distinct only in ori- 
entation, the direction is unique.

\subsubsection{Feature selection}

After building the feature vector and evaluating the best results, different texture descriptors were combined to build a hybrid texture descriptor. Afterward, feature selection was performed to simplify the prediction model by eliminating unnecessary features. Therefore, the required computational cost could decreased and the performance of the clustering algorithm be improved.

For feature selection, the gain ratio information algorithm (Raileanu and Stoffel, 2004) was used. This algorithm is a filter that overvalues features with multiple values (Quinlan, 1986). Therefore, it selects features that maximize the information gain while minimizing the number of values of an attribute.

The gain ratio information approach is resilient and combines various methods of research and evaluation. Here, the Ranker technique was used to rank the attributes based on their evaluations. In this technique, a score is used for each attribute. Therefore, a ranking is constructed according to the degree of attribute relevance. Here, 24 tests were performed using 1, 2, 3, 4 and $5 \%$, and from 10 to $100 \%$, according to steps of $5 \%$, of the feature vector length.

\subsubsection{Seeded fuzzy C-means}

SFc-means (Santos et al. 2018), is a semi-supervised clustering algorithm based on the fuzzy C-means algorithm (Li et al. 2018). In addition to the use of seeds, a clustering threshold was included, so the SFc-means algorithm does not use the original concept of centroids. Instead, the algorithm uses all available labeled examples, i.e., all the seeds, as group representations.

During clustering, the SFc-means algorithm measures the membership degree of each input sample for each seed. Then, it stores the results in a descending sequence in the membership array, as shown in Figure 8.

Another feature of the SFc-means algorithm is the establishing of a clustering threshold value $(t)$, which is responsible for the association, or the lack of association, of an input sample with a cluster. For example, in $8(a)$, the investigated superpixel is associated with the lesion group, whereas in Figure 8(b), it is not.

In this work, as we intended that all superpixels be clustered in the input image, we chose to not use a $t$. Thus, we increased the risk of superpixels being erroneously grouped. However, the included post-processing step proved to be efficient in correcting the eventual errors of the SFc-means algorithm caused by not using the threshold.

Figure 9 shows the results of the SFc-means algorithm for grouping the superpixels presented in an input image without using a cluster threshold.

\subsection{Post-processing}

Although the SFc-means clustering algorithm has a high degree of certainty, it can lead to errors, as shown in Figure 10(a) For this reason, a post-processing step is applied using mathematical morphology techniques (MM) and a geodesic active contour (GAC).

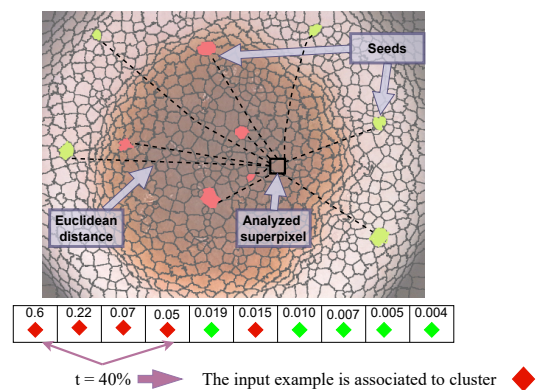

(a) Labeled data.

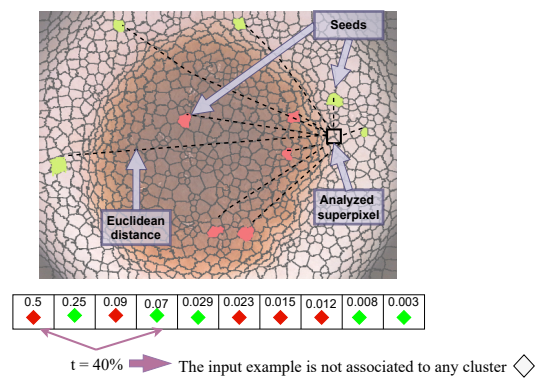

(b) Unlabeled data.

Fig. 8. Examples of thresholded results obtained using the SFc-means clustering algorithm (red indicates superpixels of the lesion area, green indicates superpixels of the image background, and the numerical values are the membership degree).

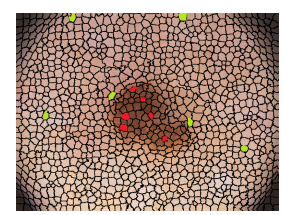

(a) Input image with $1200 \mathrm{sp}$.

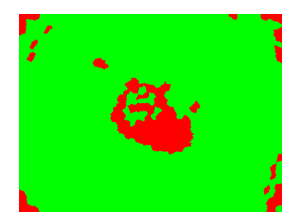

(b) Sp. of (a) after clustering.
Fig. 9. Example of a semi-supervised segmentation obtained using the SFcmeans algorithm.

An opening morphological operation is applied to eliminate the small amounts of noise that persist after the clustering step, which is then followed by an erosion. The used structuring element has a circular shape with a radius equal to $0.5 \%$ of the number of lines of the input image. The most significant connected component is then identified, as shown in Figure 10(c)

After selecting the region of the lesion under analysis, the sharp-edged fragments are removed from the image border. To do this, the convex hull of the region is calculated (Figure 10(c) and Figure 10(d) . The final contour of the lesion is then obtained by executing 100 iterations of the GAC algorithm, which was proposed by Hemalatha et al. (2018) for the segmentation of medical images, from the contour of the built convex hull (Caselles et al. 1997) (Figure 10(e)]. Thus, the GAC algorithm modifies, proportionally to the curvature, the contour of the convex hull by moving its points perpendicularly toward the border of the lesion. 


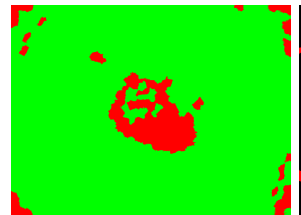

(a) Result after cluster- (b) After mathematical (c) Selection of the ing.
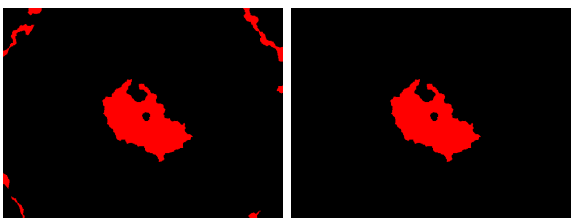
morphology.

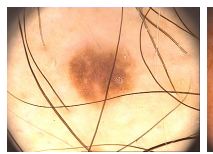

(a)

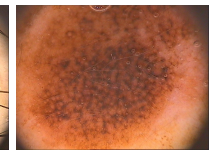

(b)

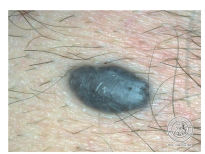

(c)

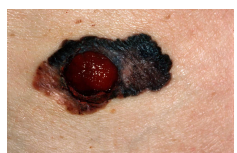

(d)
Fig. 11. Examples of images from the $\mathrm{PH}^{2}$ dataset $(\mathrm{a}, \mathrm{b})$ and from the DermIS dataset (c, d), where (a) and (c) images have benign and (b) and (d) malignant lesions.

the ISIC 2016 and ISIC 2017 datasets, we used the images from the testing phases of the respective challenges; however, since the testing phase ground truth images are not public available for the ISIC 2018 dataset, only the images from this dataset belonging to the training set were used as in Al-Masni et al. (2020); Lei et al. (2020); Tong et al. (2021); Arora et al. (2021); Qamar et al. (2021). It should be noticed that the images included in the ISIC 2016, 2017 and 2018 datasets are particularly challenging due to their high heterogeneity.

\section{proposed method.}

\section{Experiments}

This section describes the used datasets and demonstrates the performance of the proposed segmentation method.

\subsection{Image datasets}

One of the difficulties in segmenting dermoscopic images arises when the image dataset to be segmented is not homogeneous, for example, due to images collected using different cameras, from patients of different skin colors, and according to different resolutions. Therefore, numerous researchers have evaluated their systems using only one homogeneous dataset. In this study, the used image datasets were divided into development and performance datasets.

The development dataset includes images from the $\mathrm{PH}^{2}$ (Mendonça et al. 2013) dataset, which have a resolution of $768 \times 560$ pixels, and DermIS (Diepgen and Yihune, 2012) dataset, whose images have resolutions of $550 \times 360,1078 \times$ 1605 or $1,640 \times 1,043$ pixels. Table 2 indicates the number of images present in the development dataset, and Figure 11 shows examples of these images. The development dataset was used for tuning the parameters of the proposed method.

Table 2. Number of images included in the development dataset.

\begin{tabular}{cccc}
\hline Dataset & Benign & Malignant & Total \\
\hline $\mathrm{PH}^{2}$ & 160 & 40 & 200 \\
DermIS & 87 & 114 & 201 \\
\hline Total & 247 & 154 & 401 \\
\hline
\end{tabular}

To verify that the proposed method can be successfully used in broad heterogeneous images, it was applied to the images in the performance dataset, which is composed of images from the ISIC 2016 (Gutman et al. 2016), ISIC 2017 (Codella et al. 2017) and ISIC 2018 (Codella et al., 2018) datasets with resolution raging from $767 \times 576$ to $6682 \times 4401$ pixels. Table 3 indicates the number of images included in the performance dataset, and Figure 12 shows examples of these images. As to
Table 3. Number of images included in the performance dataset (the ISIC 2018 dataset has no information about the lesions being malignant or benign).

\begin{tabular}{cccc}
\hline Dataset & Benign & Malignant & Total \\
\hline ISIC 2016 & 304 & 75 & 379 \\
ISIC 2017 & 483 & 117 & 600 \\
ISIC 2018 & - & - & 2,594 \\
\hline Total & - & - & 3,573 \\
\hline
\end{tabular}

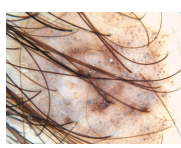

(a)

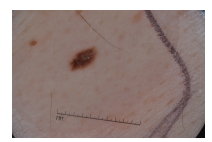

(d)

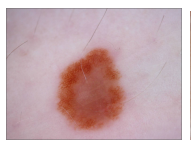

(b)

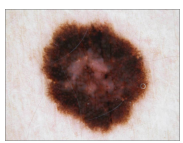

(e)

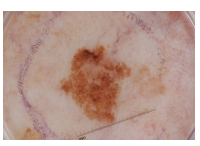

(c)

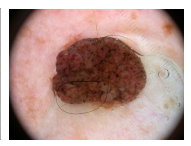

(f)
Fig. 12. Examples of images from the ISIC 2016 (a, b), ISIC 2017 (c, d), and ISIC 2018 (e, f) datasets where (a) and (c) are benign lesions, (b) and (d) are malignant lesions, and (e) and (f) are undefined.

\subsection{Evaluation metrics}

In this study, the term 'positive' is used to designate lesion areas, and 'negative' to designate non-lesion areas. To assess the quality of the obtained segmentation results, the following quality metrics were used: accuracy $(A c c)$, specificity $(S p e)$, sensitivity ( $\mathrm{Sen}$ ) and Jaccard index ( $\mathrm{Jac}$ ), which were computed as:

$$
\begin{gathered}
A c c=\frac{T P+T N}{T P+T N+F P+F N}, \\
S p e=\frac{T N}{T N+F P},
\end{gathered}
$$




$$
\begin{gathered}
\text { Sen }=\frac{T P}{T P+F N}, \\
J a c=\frac{T P}{T P+F P+F N}
\end{gathered}
$$

where $T P$, means True Positive, $T N$, True Negative, $F P$, False Positive and $F N$, False Negative.

Codella et al. (2018) demonstrated that the direct use of the Jaccard index as a measure of segmentation performance does not accurately reflect the number of images where the computational segmentation fails or falls outside the expert interobserver variability. Therefore, it is more suitable to use the threshold Jaccard index metric $(T J I)$.

In the ISIC challenge of 2018, the organizers chose 0.65 as the a basis value for the threshold Jaccard index metric to indicate segmentation failure for a skin lesion image. To compute the $T J I$ metric, a score for each image $(i)$ is calculated based on the Jaccard index:

$$
\left\{\begin{array}{c}
\text { score }_{i}=0, \text { if } J a c<0.65, \\
\text { score }_{i}=J a c, \text { otherwise. }
\end{array}\right.
$$

Given a dataset with $n$ images, the final $T J I$ value is defined as the mean of all the per-image scores:

$$
T J I=\frac{\sum_{i=1}^{n} \text { score }_{i}}{n} .
$$

\subsection{Results}

The proposed method requires the use of four parameters: 1) the number of superpixels to be generated by the SLICO algorithm - 400, 600,800,1000, 1200, 1400 or 1600; 2) color channels to use for feature extraction - RGB, $\mathrm{L}^{*} \mathrm{a}^{*} \mathrm{~b}^{*}$ or RGB $+\mathrm{L}^{*} \mathrm{a}^{*} \mathrm{~b}^{*}$; 3) texture descriptors to use - GLCM, GLRLM, Geostatistics, LBP, Tamura or combinations of these descriptors; and 4) the percentage of the most significant features to use.

To find the best setting of each parameter, the method was performed ten times. For each execution, the seeds were chosen randomly; this choice was made to simulate the different choices made by users, i.e. physicians. Additionally, TJI was multiplied by 100 to homogenize the visualization and to facilitate the understanding of the tables presented here. Table 4 presents the averages and standard deviations of the best results obtained by the proposed method on the development dataset.

When $T J I$ was used as the primary segmentation performance metric, a hybrid mixture of the RGB and $\mathrm{L}^{*} \mathrm{a} * \mathrm{~b} *$ color channels yielded the six best combinations, as can be verified in Table 4. Three values for the number of superpixels (1000, 1200 and 1400) and two descriptors (GLCM and Tamura) were found to be optimal. To set up the hybrid texture feature vector, the settings that yielded the best results were combined. Therefore, the GLCM and Tamura descriptors were concatenated to build three additional feature vectors, using 1000 superpixels in the RGB and L*a* b* color channels. A similar setup was then applied using 1200 and 1400 superpixels.

Empirical tests were performed to define the dimensionality

\begin{tabular}{|c|c|c|c|c|c|c|}
\hline \multicolumn{7}{|c|}{ GLCM } \\
\hline$N^{\circ}$ of Sp. & Color Channel & $\operatorname{Acc}(\%)$ & Spe $(\%)$ & $\operatorname{Sen}(\%)$ & $\operatorname{Jac}(\%)$ & $T J I(\%)$ \\
\hline 1200 & $\mathrm{RGB}+\mathrm{L} * \mathrm{a} * \mathrm{~b} *$ & $97.02 \pm 0.06$ & $96.50 \pm 015$ & $80.83 \pm 0.36$ & $77.42 \pm 0.30$ & $\overline{84.12} \pm 0.01$ \\
\hline 1400 & $\mathrm{RGB}+\mathrm{L} * \mathrm{a} * \mathrm{~b} *$ & $97.12 \pm 0.11$ & $96.63 \pm 0.22$ & $81.66 \pm 0.67$ & $78.43 \pm 0.35$ & $\mathbf{8 3 . 7 8} \pm 0.02$ \\
\hline 1000 & $\mathrm{RGB}+\mathrm{L} * \mathrm{a} * \mathrm{~b} *$ & $96.56 \pm 0.06$ & $96.15 \pm 0.77$ & $78.70 \pm 0.88$ & $75.26 \pm 0.70$ & $\mathbf{8 1 . 7 9} \pm 0.01$ \\
\hline 800 & $\mathrm{~L} * \mathrm{a} * \mathrm{~b} *$ & $96.83 \pm 0.08$ & $96.52 \pm 0.30$ & $79.80 \pm 0.69$ & $76.27 \pm 0.54$ & $81.69 \pm 0.01$ \\
\hline \multicolumn{7}{|c|}{ GLRLM } \\
\hline$N^{\circ}$ of Sp. & Color Channel & $A c c(\%)$ & Spe(\%) & Sen $(\%)$ & $J a c(\%)$ & $T J I(\%)$ \\
\hline 600 & RGB & $94.94 \pm 0.19$ & $94.22 \pm 0.58$ & $74.25 \pm 0.94$ & $69.19 \pm 0.66$ & $70.62 \pm 0.02$ \\
\hline 1000 & RGB & $95.33 \pm 0.15$ & $94.85 \pm 0.09$ & $73.11 \pm 1.15$ & $68.96 \pm 1.02$ & $70.29 \pm 0.02$ \\
\hline 1200 & RGB & $95.47 \pm 0.09$ & $95.17 \pm 0.21$ & $72.84 \pm 0.39$ & $69.01 \pm 0.41$ & $68.94 \pm 0.01$ \\
\hline 1400 & RGB & $94.81 \pm 0.10$ & $95.27 \pm 0.25$ & $66.25 \pm 0.66$ & $62.86 \pm 0.69$ & $63.90 \pm 0.01$ \\
\hline \multicolumn{7}{|c|}{ Geostatistics } \\
\hline$N^{\circ}$ of Sp. & Color Channel & $\operatorname{Acc}(\%)$ & Spe(\%) & Sen $(\%)$ & $J a c(\%)$ & $T J I(\%)$ \\
\hline 1400 & $\mathrm{RGB}+\mathrm{L} * \mathrm{a}^{*} \mathrm{~b}^{*}$ & $94.05 \pm 0.15$ & $93.18 \pm 0.36$ & $73.54 \pm 0.51$ & $66.30 \pm 0.30$ & $67.11 \pm 0.01$ \\
\hline 1200 & $\mathrm{~L} * \mathrm{a} * \mathrm{~b} *$ & $94.18 \pm 0.20$ & $93.28 \pm 0.44$ & $72.35 \pm 0.28$ & $64.95 \pm 0.27$ & $66.52 \pm 0.02$ \\
\hline 1200 & $\mathrm{RGB}+\mathrm{L} * \mathrm{a} * \mathrm{~b} *$ & $93.92 \pm 0.27$ & $93.06 \pm 0.54$ & $71.96 \pm 0.30$ & $65.12 \pm 0.32$ & $65.36 \pm 0.01$ \\
\hline 1400 & RGB & $93.78 \pm 0.24$ & $92.88 \pm 0.50$ & $72.07 \pm 0.86$ & $64.85 \pm 0.48$ & $65.19 \pm 0.02$ \\
\hline \multicolumn{7}{|c|}{ LBP } \\
\hline$N^{\circ}$ of $\mathrm{Sp}$. & Color Channel & $A c c(\%)$ & Spe(\%) & $\operatorname{Sen}(\%)$ & $J a c(\%)$ & $T J I(\%)$ \\
\hline 600 & $\mathrm{~L} * \mathrm{a} * \mathrm{~b} *$ & $91.30 \pm 0.20$ & $93.37 \pm 0.42$ & $44.87 \pm 1.08$ & $40.57 \pm 1.07$ & $32.95 \pm 0.02$ \\
\hline 800 & $L * a * b *$ & $91.17 \pm 0.24$ & $93.63 \pm 0.39$ & $42.74 \pm 1.97$ & $38.89 \pm 1.82$ & $30.28 \pm 0.02$ \\
\hline 600 & $\mathrm{RGB}+\mathrm{L} * \mathrm{a} * \mathrm{~b} *$ & $90.84 \pm 0.19$ & $93.44 \pm 0.40$ & $42.09 \pm 1.10$ & $37.90 \pm 1.11$ & $29.37 \pm 0.02$ \\
\hline 1000 & $\mathrm{~L} * \mathrm{a} * \mathrm{~b}^{*}$ & $90.87 \pm 0.13$ & $93.58 \pm 0.21$ & $40.70 \pm 0.58$ & $37.14 \pm 0.53$ & $28.12 \pm 0.01$ \\
\hline \multicolumn{7}{|c|}{ Tamura } \\
\hline$N^{\circ}$ of Sp. & Color Channel & $\operatorname{Acc}(\%)$ & Spe $(\%)$ & $\operatorname{Sen}(\%)$ & $\operatorname{Jac}(\%)$ & $T J I(\%)$ \\
\hline 1400 & $\mathrm{RGB}+\mathrm{L}^{*} \mathrm{a} * \mathrm{~b}^{*}$ & $97.20 \pm 0.11$ & $96.81 \pm 0.14$ & $82.01 \pm 0.95$ & $78.68 \pm 0.98$ & $\mathbf{8 4 . 3 7 \pm 0 . 0 1}$ \\
\hline 1000 & $\mathrm{RGB}+\mathrm{L} * \mathrm{a} * \mathrm{~b} *$ & $97.03 \pm 0.11$ & $96.57 \pm 0.09$ & $81.27 \pm 1.03$ & $77.86 \pm 0.98$ & $\mathbf{8 4 . 3 6} \pm 0.02$ \\
\hline 1200 & $\mathrm{RGB}+\mathrm{L} * \mathrm{a} * \mathrm{~b} *$ & $97.16 \pm 0.06$ & $96.60 \pm 0.20$ & $82.09 \pm 1.06$ & $78.70 \pm 0.90$ & $\mathbf{8 3 . 7 0} \pm 0.01$ \\
\hline 1200 & RGB & $96.94 \pm 0.08$ & $96.90 \pm 0.15$ & $77.10 \pm 1.11$ & $74.14 \pm 0.99$ & $81.76 \pm 0.01$ \\
\hline
\end{tabular}
of the definitive feature vector, always having in mind a good
Table 4. Best segmentation results obtained by the proposed method on the development dataset. (Best values are in bold.)

compromise between $T J I$ and the vector length. In these tests, the gain ratio algorithm was applied, and the vectors built were assembled with the features ranked in the descending order of the gain ratio. When the experiments were performed, these ranked features were used, starting from the highest gain ratio and adding the other features in order to include all of them. Thus, it was used feature vectors with the percentage of the most significant features in a sum of 216 tests, i.e., 24 feature vectors with different lengths multiplied by nine descriptors. Figure 13 depicts the results obtained from these experiments, and Table 5 presents the numbers of attributes that led to the best results.

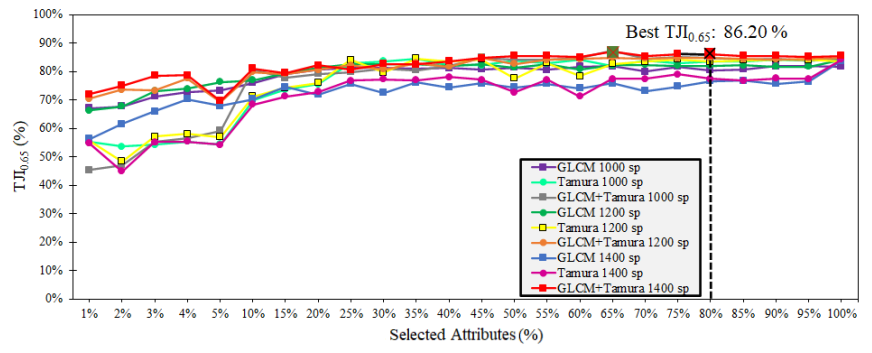

Fig. 13. Values obtained for $T J I$ using different numbers of features, sorted by gain ratio.

Using $T J I$ as the most relevant segmentation metric, the best results were obtained with 1,400 superpixels and $80 \%$ of the GLCM and Tamura features, as indicated in Table 5

Figure 14 depicts the feature percentage chosen from the descriptors and the vector positions sorted by the gain ratio algorithm in the GLCM + Tamura feature vector. This figure shows 
Table 5. Best achieved results in the feature selection step. (Best values are in bold.)

\begin{tabular}{|c|c|c|c|c|c|c|c|c|}
\hline \multicolumn{9}{|c|}{ Using 1000 superpixels } \\
\hline Descriptor(s) & $\operatorname{Data}(\%)$ & $\begin{array}{l}N^{\circ} \\
\text { Attributes }\end{array}$ & of & $\operatorname{Acc}(\%)$ & Spe(\%) & $\operatorname{Sen}(\%)$ & $\operatorname{Jac}(\%)$ & $T J I(\%)$ \\
\hline GLCM & 90 & 92 & & $96.88 \pm 0.05$ & $96.43 \pm 0.11$ & $79.65 \pm 0.77$ & $76.26 \pm 0.71$ & $81.94 \pm 0.01$ \\
\hline Tamura & 90 & 17 & & $97.02 \pm 0.04$ & $\mathbf{9 6 . 5 6} \pm 0.08$ & $81.19 \pm 0.73$ & $77.73 \pm 0.69$ & $84.37 \pm 0.01$ \\
\hline GLCM + Tamura & 80 & 96 & & $\mathbf{9 7 . 0 4} \pm 0.06$ & $96.53 \pm 0.15$ & $\mathbf{8 2 . 2 7} \pm 0.63$ & $\mathbf{7 8 . 8 2} \pm 0.63$ & $\mathbf{8 4 . 8 7} \pm 0.01$ \\
\hline \multicolumn{9}{|c|}{ Using 1200 superpixels } \\
\hline Descriptor(s) & $\operatorname{Data}(\%)$ & $\begin{array}{l}N^{\circ} \\
\text { Attributes }\end{array}$ & of & $\operatorname{Acc}(\%)$ & Spe(\%) & $\operatorname{Sen}(\%)$ & $\operatorname{Jac}(\%)$ & $T J I(\%)$ \\
\hline GLCM & 100 & 102 & & $97.02 \pm 0.07$ & $96.50 \pm 0.15$ & $80.83 \pm 0.43$ & $77.42 \pm 0.41$ & $84.12 \pm 0.02$ \\
\hline Tamura & 90 & 17 & & $\mathbf{9 7 . 1 3} \pm 0.08$ & $96.68 \pm 0.12$ & $\mathbf{8 1 . 7 2} \pm 0.65$ & $\mathbf{7 8 . 3 4} \pm 0.74$ & $84.29 \pm 0.01$ \\
\hline GLCM + Tamura & 100 & 120 & & $97.12 \pm 0.08$ & $96.79 \pm 0.18$ & $81.16 \pm 0.48$ & $78.05 \pm 0.44$ & $\mathbf{8 4 . 9 6} \pm 0.03$ \\
\hline \multicolumn{9}{|c|}{ Using 1400 superpixels } \\
\hline Descriptor(s) & $\operatorname{Data}(\%)$ & $\begin{array}{l}N^{\circ} \\
\text { Attributes }\end{array}$ & of & $\operatorname{Acc}(\%)$ & Spe(\%) & $\operatorname{Sen}(\%)$ & $\operatorname{Jac}(\%)$ & $T J I(\%)$ \\
\hline GLCM & 100 & 102 & & $97.13 \pm 0.06$ & $96.63 \pm 0.19$ & $82.66 \pm 0.45$ & $78.43 \pm 0.33$ & $83.78 \pm 0.01$ \\
\hline Tamura & 100 & 18 & & $\mathbf{9 7 . 2 0} \pm 0.07$ & $\mathbf{9 6 . 8 1} \pm 0.21$ & $82.01 \pm 0.23$ & $78.68 \pm 0.13$ & $84.36 \pm 0.02$ \\
\hline GLCM + Tamura & 80 & 96 & & $97.18 \pm 0.08$ & $96.66 \pm 0.10$ & $\mathbf{8 2 . 1 1} \pm 0.89$ & $\mathbf{7 8 . 9 4} \pm 0.72$ & $\mathbf{8 6 . 2 0} \pm 0.01$ \\
\hline
\end{tabular}

that the GLCM descriptor obtained the best rate on the crucial feature subset.

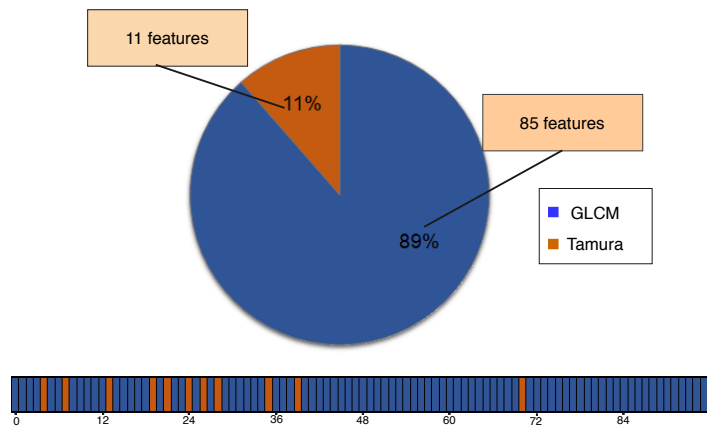

Fig. 14. Origin of the 96 selected features (the bar at the bottom indicates the source of the characteristics at each position).

The last row of Table 5 presents the results obtained by the proposed method using a heuristic search with the best parameters. To demonstrate that the proposed method performs well on images from different datasets, it was applied to the performance dataset, which includes images of the ISIC 2016 and ISIC 2017 datasets, using the best parameters found in the previous step. The obtained results and the average processing times, in seconds, are indicated in Table 6 The tests were carried out on a computer with an Intel Core i7-3630QM CPU and 8GB of RAM, using MATLAB MATLAB (2010).

Table 6. Average and standard deviation of the segmentation quality metrics obtained by the proposed method on the performance dataset and the respective average processing times.

\begin{tabular}{lllllll}
\hline Dataset & Acc $(\%)$ & $S p e(\%)$ & $S e n(\%)$ & $\operatorname{Jac}(\%)$ & $T J I(\%)$ & Time(s) \\
\hline ISIC 2016 & $96.24 \pm 0.08$ & $95.62 \pm 0.22$ & $86.12 \pm 0.09$ & $82.43 \pm 0.16$ & $94.28 \pm 0.01$ & $4.12 \pm 0.35$ \\
ISIC 2017 & $95.80 \pm 0.05$ & $94.62 \pm 0.06$ & $78.71 \pm 0.23$ & $74.13 \pm 0.29$ & $78.97 \pm 0.02$ & $4.75 \pm 0.40$ \\
ISIC 2018 & $97.08 \pm 0.13$ & $97.42 \pm 0.15$ & $93.48 \pm 0.89$ & $89.89 \pm 0.58$ & $99.67 \pm 0.01$ & $5.19 \pm 0.55$
\end{tabular}

The values presented in Table 6 indicate that the proposed method achieved an accuracy greater than $95.80 \%$ for ISIC 2016, ISIC 2017 and ISIC 2018 datasets, with a peak of $97.8 \%$ in the ISIC 2018 dataset, respectively. Additionally, the Jac- card index values indicate that, on average, the similarity between the segmented regions and their ground truths was higher than $74 \%$ in the three datasets, with a peak of $89.89 \%$ for the ISIC 2018 dataset. These results reflect the values obtained for $T J I$, which indicates that, in $94.28 \%$ of the ISIC 2016 dataset and $99.67 \%$ of the ISIC 2018 dataset images, the agreement was higher than $65 \%$ between the segmented regions and their ground truth. The ISIC 2017 dataset is a particularly challenging case; even though, a TJI of $78.97 \%$ was achieved by the proposed method in this dataset. It can be noticed that the proposed method took, on average, between 4 and 6 seconds to execute with a very small standard deviation, which suggests that the execution time is relatively insensitive to the size of the input image .

Skin lesion segmentation algorithms can assist specialists in extracting information related to the $\mathrm{ABCD}$ rule from the input images, for example, or in producing inputs for lesion classification systems. Therefore, it is essential to determine their effectiveness of segmentation for benign and malignant lesions. Table 7 presents the effectiveness of the proposed segmentation method for images containing benign and malignant lesions in the ISIC 2016 and ISIC 2017 datasets.

Table 7. Effectiveness of the proposed method in the segmentation of images containing benign and malignant lesions in the ISIC 2016 and ISIC 2017 datasets. (Best values are in bold.)

\begin{tabular}{llllll}
\hline \multicolumn{5}{c}{ ISIC 2016} \\
\hline Dataset & $A c c(\%)$ & $S p e(\%)$ & $\operatorname{Sen}(\%)$ & $\operatorname{Jac}(\%)$ & $T J I(\%)$ \\
\hline Benign & $\mathbf{9 6 . 5 5} \pm 0.08$ & $\mathbf{9 6 . 1 6} \pm 0.25$ & $85.08 \pm 0.08$ & $81.77 \pm 0.16$ & $93.42 \pm 0.01$ \\
Malignant & $94.99 \pm 0.06$ & $93.44 \pm 0.10$ & $\mathbf{9 0 . 3 6} \pm 0.11$ & $\mathbf{8 5 . 1 2} \pm 0.17$ & $\mathbf{9 7 . 7 8} \pm 0.02$ \\
\hline \multicolumn{5}{c}{ ISIC 2017} \\
\hline Dataset & $A c c(\%)$ & $S p e(\%)$ & $S e n(\%)$ & $\operatorname{Jac}(\%)$ & $T J I(\%)$ \\
\hline Benign & $\mathbf{9 6 . 2 4} \pm 0.04$ & $\mathbf{9 5 . 3 7} \pm 0.06$ & $77.92 \pm 0.21$ & $73.80 \pm 0.28$ & $78.42 \pm 0.02$ \\
Malignant & $93.98 \pm 0.07$ & $91.52 \pm 0.07$ & $\mathbf{8 1 . 9 6} \pm 0.29$ & $\mathbf{7 5 . 5 3} \pm 0.32$ & $\mathbf{8 1 . 2 0} \pm 0.03$ \\
\hline
\end{tabular}

The results presented in Table 7 suggest that the proposed method achieved somewhat similar evaluation rates. Therefore, one can conclude that it performed satisfactorily well for benign and malignant images. Malignant lesions have the characteristic of having different colors and, therefore, their region can be 
easily mistaken with the background, for example. However, our method has proven to be able to overcome this challenge. First, texture characteristics are used as input to the clustering algorithm. Although there is the possibility of similar colors, skin regions are usually more homogeneous. Second, all the post-processing step is based on the size and position of the regions pre-segmented by the cluster algorithm, and only connected segmented regions are considered as belonging to the lesion under analysis. By observing the standard deviations presented in Table 7) one can realize that the choice of the used seeds has low impact of the segmentation success rates.

Figure 15 depicts the results of the proposed segmentation method overlapped by the corresponding ground truths. For each individual dataset included in the performance dataset. Particularly, this figure shows the cases corresponding to the worst (Figures 15(a), 15(d), and 15(g)), the median (Figures 15(b) 15(e), and 15(h) , and the best (Figures 15(c), 15(f), and 15(i) values found for the Jaccard index.

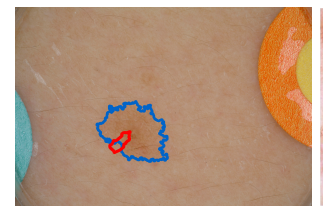

(a) $\mathrm{Jac}=3.97 \%$

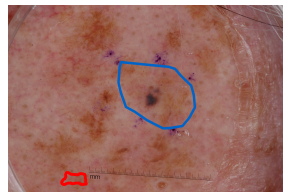

(d) $J a c=0 \%$

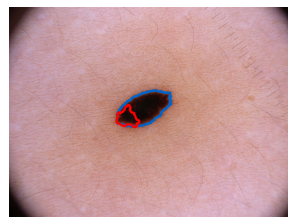

(g) $J a c=20.58 \%$

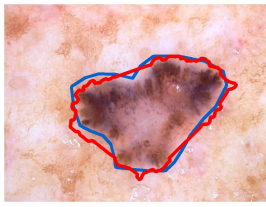

(b) Jac $=87.14 \%$

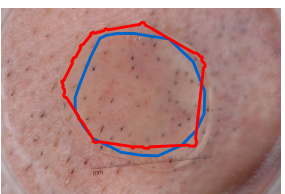

(e) $J a c=82.25 \%$

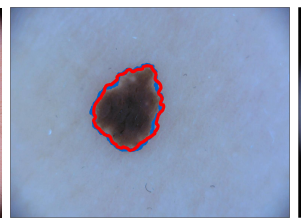

(h) $\mathrm{Jac}=91.73 \%$

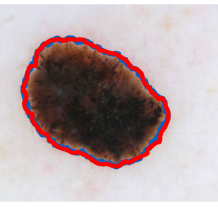

(c) $\mathrm{Jac}=97.09 \%$

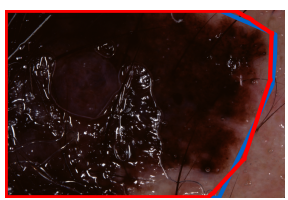

(f) $\mathrm{Jac}=97.84 \%$

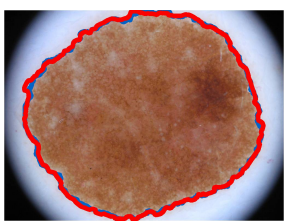

(i) $\mathrm{Jac}=98.16 \%$
Fig. 15. Examples of skin lesions from the ISIC 2016 (a, b, c), ISIC 2017 (d, e, f) and ISIC 2018 (g, h, i) datasets with the segmentations obtained by the proposed method (in red) and the corresponding ground truths (in blue) overlapped.

Additionally, Table 8 presents the average of the results obtained by the proposed method in 33 images of the ISIC 2018 test set. This assessment was possible because a dermatologist from the Department of Specialized Medicine at the Federal University of Piauí, in Brazil, delineated the ground truth of these 33 images. Figure 16 shows the cases corresponding to the worst (Figure 16(a)), the median (Figure $16(\mathrm{~b})]$, and the best (Figure 16(c) values found for the Jaccard index.

The results presented in Table 8 show that the proposed method achieved an accuracy of $97.20 \%$ with an average of 3.11 seconds of processing time. These values are similar to those obtained in the previous tests. A Jaccard index of 79.57\% demonstrates that the ISIC 2018 test set is as challenging as the
Table 8. Average and standard deviation of the segmentation quality metrics obtained by the proposed method in a subset of the ISIC 2018 test dataset and the respective average processing times.

\begin{tabular}{llllll}
\hline$A c c(\%)$ & S pe $(\%)$ & Sen $(\%)$ & $\operatorname{Jac}(\%)$ & TJI $(\%)$ & Time(s) \\
\hline $97.20 \pm 0.11$ & $98.09 \pm 0.04$ & $86.91 \pm 1.72$ & $79.57 \pm 1.50$ & $91.92 \pm 0.06$ & $3.11 \pm 1.91$ \\
\hline
\end{tabular}

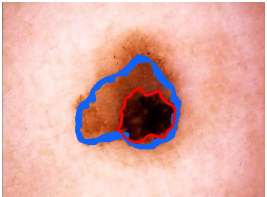

(a) $J a c=50.96 \%$

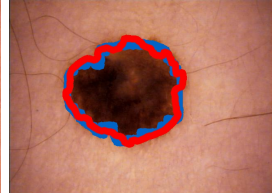

(b) $\mathrm{Jac}=82.11 \%$

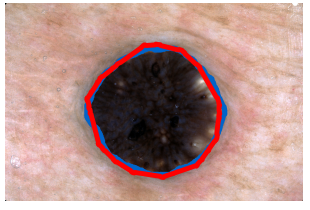

(c) $\mathrm{Jac}=93.10 \%$
Fig. 16. Examples of skin lesions from the ISIC 2018 test dataset with the segmentations obtained by the proposed method (in red) and the corresponding ground truths (in blue) overlapped.

DermIS and ISIC 2017 datasets. However, even with this difficulty, a $T J I$ of $91.92 \%$ indicates that most images were segmented approximately to the delineations made by the specialist.

\section{Discussion}

The results obtained in this study are equivalent to those found in the state-of-the-art. Some of the current studies used private-labeled datasets, and therefore, it is not easy to reproduce their results. However, because the $\mathrm{PH}^{2}$, DermIS, ISIC 2016 and ISIC 2017 datasets are publicly available, a comparison with state-of-the-art methods that also used these datasets was possible. Table 9 presents the results of this comparison as to the development dataset, with the values indicated for the existing related methods been obtained from their original articles.

For the $\mathrm{PH}^{2}$ dataset, the accuracy obtained by the proposed method was lower than those reported by Xie et al. (2020) and Araújo et al. (2021), with a difference of $0.4 \%$ and of $0.7 \%$, respectively. The obtained Jaccard values were lower than those reported by Xie et al. (2020), with a difference of only $1.76 \%$. For the DermIS dataset, the accuracy of the proposed method was $1.62 \%$ lower than the one reported by Filali and Belkadi (2019) and 2.06\% lower than the one reported by Araújo et al. (2021). As could be realized, the differences were not statistically significant in the examples where the proposed method had a poorer average performance. Notably, although the proposed method attained a high accuracy in the DermIS dataset, the value of $73.63 \%$ for $T J I$ indicates that it can be improved.

Table 10 presents the results of the comparison between the proposed method and related ones as to the performance dataset.

For the ISIC 2016 dataset, the proposed method achieved higher accuracy than the studied state-of-art methods. The value obtained by the proposed method for Jac was higher than the ones obtained by the other methods, except for that of Nida et al. (2019) and Tong et al. (2021). For the ISIC 2017 dataset, in terms of accuracy, the proposed method exceeds all the other methods under comparison; however, the Jac value was only 
Table 9. Comparison of the results obtained by the proposed method with those obtained by state-of-the-art methods as to the development dataset. (Best valued are in bold.)

\begin{tabular}{|c|c|c|c|c|c|}
\hline \multicolumn{6}{|c|}{$\mathrm{PH}^{2}$} \\
\hline Method & $\operatorname{Acc}(\%)$ & Spe(\%) & $\operatorname{Sen}(\%)$ & $\operatorname{Jac}(\%)$ & $T J I(\%)$ \\
\hline Khalid et al. (2016) & 93.87 & - & - & - & - \\
\hline Pennisi et al. 2016 & 89.66 & 97.22 & 80.24 & - & - \\
\hline \begin{tabular}{|lll} 
Ünver and Ayan \\
$2019 \mid$
\end{tabular} & 92.99 & 94.02 & 83.63 & 79.54 & - \\
\hline $\begin{array}{l}\text { Vasconcelos et al. } \\
2019\end{array}$ & 94.59 & 97.99 & 91.72 & 86.16 & - \\
\hline Goyal et al. 2020 & 93.80 & 92.90 & 93.20 & 83.90 & - \\
\hline Xie et al. $\{2020\}$ & 96.50 & 94.60 & 96.70 & 89.40 & _- \\
\hline Pereira et al. 2020 & - & - & - & 86.30 & - \\
\hline Tong et al. $\{2021\}$ & 94.30 & 93.70 & 96.00 & 84.20 & - \\
\hline Araújo et al. (2021) & 96.80 & 97.60 & 94.30 & 87.30 & - \\
\hline Proposed Method & $96.10 \pm 0.06$ & $93.72 \pm 0.17$ & $91.93 \pm 0.38$ & $87.64 \pm 0.26$ & $\mathbf{9 8 . 8 3} \pm 0.00$ \\
\hline \multicolumn{6}{|c|}{ DermIS } \\
\hline Method & $A c c(\%)$ & Spe(\%) & Sen $(\%)$ & $\operatorname{Jac}(\%)$ & $T J I(\%)$ \\
\hline $\begin{array}{l}\text { Filali and Belkadi } \\
2019\end{array}$ & 97.86 & 99.00 & 91.86 & - & - \\
\hline Araújo et al. 2021 & 98.30 & 98.70 & 92.30 & 77.44 & - \\
\hline Proposed Method & $96.24 \pm 0.08$ & $\mathbf{9 9 . 5 8} \pm 0.05$ & $72.33 \pm 1.49$ & $70.28 \pm 1.28$ & $73.63 \pm 0.03$ \\
\hline
\end{tabular}

Table 10. Comparison of the results obtained by the proposed method with those obtained by state-of-the-art methods as to the performance dataset. (Best valued are in bold.)

\begin{tabular}{|c|c|c|c|c|c|}
\hline \multicolumn{6}{|c|}{ ISIC 2016} \\
\hline Method & $\operatorname{Acc}(\%)$ & Spe $(\%)$ & $\operatorname{Sen}(\%)$ & $\operatorname{Jac}(\%)$ & $T J I(\%)$ \\
\hline Nida et al. (2019) & 94.20 & 94.00 & 95.00 & 93.00 & - \\
\hline Garcia-Arroyo and & 93.40 & 97.80 & 87.00 & 79.10 & - \\
\hline $\begin{array}{l}\text { Garcia-Zapirain } \\
|2019|\end{array}$ & & & & & \\
\hline $\begin{array}{lll}\text { Al-Masni et } & \text { al. } \\
2020 & & \end{array}$ & 81.79 & 71.40 & 81.80 & - & - \\
\hline Le1 et al. 2020 & 96.00 & 96.80 & 93.70 & - & 87.10 \\
\hline Tong et al. (2021) & 95.40 & 96.10 & 92.70 & 84.50 & - \\
\hline Proposed Method & $\mathbf{9 6 . 2 4} \pm 0.08$ & $95.62 \pm 0.22$ & $86.12 \pm 0.09$ & $82.43 \pm 0.16$ & $\mathbf{9 4 . 2 8} \pm 0.01$ \\
\hline \multicolumn{6}{|c|}{ ISIC 2017} \\
\hline Method & $\operatorname{Acc}(\%)$ & Spe(\%) & $\operatorname{Sen}(\%)$ & $\operatorname{Jac}(\%)$ & $T J I(\%)$ \\
\hline Li et al. (2018) & 94.30 & 95.30 & 87.90 & 79.80 & - \\
\hline L1 and Shen (2018) & 95.00 & 97.40 & 85.50 & 75.30 & - \\
\hline \begin{tabular}{|lll} 
Unver and Ayan \\
2019
\end{tabular} & 93.39 & 92.68 & 90.82 & 74.81 & - \\
\hline \begin{tabular}{|l|l|} 
Fulgencio & Navarro \\
and Bescós $\mid\{2019\}$
\end{tabular} & 95.50 & - & - & 76.90 & - \\
\hline $\begin{array}{l}\text { Garcia-Arroyo and } \\
\text { Garcia-Zapirain } \\
2019\end{array}$ & 88.40 & 92.30 & 86.90 & 66.50 & - \\
\hline Goyal et al. 2020 & 94.08 & 95.00 & 89.93 & 79.34 & - \\
\hline Xie et al. $\{2020\}$ & 94.70 & 96.80 & 87.40 & 80.40 & - \\
\hline $\begin{array}{lll}\text { Al-Masni et } & \text { al. } \\
2020 & & \end{array}$ & 81.57 & 80.62 & 75.33 & - & - \\
\hline Le1 et al. 2020 & 93.50 & 97.60 & 83.50 & - & 77.10 \\
\hline Tong et al. (2021) & 92.60 & 96.50 & 82.50 & 74.20 & - \\
\hline Proposed Method & $\mathbf{9 5 . 8 0} \pm 0.05$ & $94.62 \pm 0.06$ & $78.71 \pm 0.23$ & $74.13 \pm 0.29$ & $\mathbf{7 8 . 9 7} \pm 0.02$ \\
\hline \multicolumn{6}{|c|}{ ISIC 2018} \\
\hline Method & $\operatorname{Acc}(\%)$ & Spe(\%) & $\operatorname{Sen}(\%)$ & $J a c(\%)$ & $T J I(\%)$ \\
\hline Arora et al. $[2021)$ & 95.00 & 95.00 & 94.00 & 83.00 & - \\
\hline Le1 et al. $\mid 2020\}$ & 92.90 & 91.10 & 95.30 & - & 82.40 \\
\hline Qamar et al. (2021) & 96.25 & 97.00 & 96.50 & 83.30 & - \\
\hline Proposed Method & $\mathbf{9 7 . 0 8} \pm 0.13$ & $\mathbf{9 7 . 4 2} \pm 0.15$ & $93.48 \pm 0.89$ & $89.89 \pm 0.58$ & $99.67 \pm 0.01$ \\
\hline
\end{tabular}

higher than the one obtained by Garcia-Arroyo and GarciaZapirain (2019). In the ISIC 2018 dataset, our method underperformed only for the sensitivity metric; however, it should be highlighted that it achieved a $T J I$ of $99.67 \%$, which indicates that the segmentation was effective in almost all the images of this dataset.

The state-of-the-art methods included in Table 10 used only images from the same dataset for both parameter tuning, e.g., in the training of the classification step, and performance evaluation. This means that these methods are more efficient for those pre-known images, unlike the proposed method, where the ISIC 2016, ISIC 2017 and ISIC 2018 datasets were not used in the development step. This confirms further the superior portability and robustness of the proposed segmentation method.

\section{Conclusions and Future Work}

Segmentation of skin lesions is an essential topic in the field of CAD systems for dermoscopic images. In this article, a semiautomatic method for the segmentation of skin lesions in dermoscopic images was presented. The approach is based on superpixels, texture information and semi-supervised clustering.

The proposed method requires that the user identify areas of the lesion and background in the image to be analyzed. However, these areas correspond to only $1 \%$ of the superpixels automatically generated for the input image. In practical terms, this means that a minimal number of areas are required to be identified. Further, the results achieved by the proposed method are similar to those obtained via manual segmentation, but the time required is significantly less than the one required manually.

In state-of-the-art methods, images from the same dataset are usually used to define and evaluate their parameters. However, these settings do not represent a natural application environment for the segmentation of skin lesions. Hence, in this study, to simulate an environment closer to the one found in real-world clinical scenarios, 401 images gather from the $\mathrm{PH}^{2}$ and DermIS datasets were used to establish the parameters of the proposed method. Then, the performance of the method was evaluated using 3,573 images gather from the ISIC 2016, ISIC 2017 and ISIC 2018 datasets, which confirmed the good segmentation performance and the high portability and robustness of the proposed method.

The results obtained by the proposed method are promising, but can be further improved. Therefore, we plan to automatically estimate the number of superpixels to be generated for each image under analysis and use other types of texture information. Also, we have intention to evaluate other postprocessing image techniques and perform cross-validation on the results.

\section{Acknowledgements}

This study was financed in part by the "Coordenação de Aperfeiçoamento de Pessoal de Nível Superior" (CAPES), in Brazil, Finance Code 001.

\section{References}

Abbasi, N.R., Shaw, H.M., Rigel, D.S., Friedman, R.J., McCarthy, W.H., Osman, I., Kopf, A.W., Polsky, D., 2004. Early diagnosis of cutaneous melanoma: revisiting the abcd criteria. JAMA 292, 2771-2776. URL: https://doi.org/10.1001/jama.292.22.2771 doi 10.1001/jama. 292.22.2771

Al-Masni, M.A., Kim, D.H., Kim, T.S., 2020. Multiple skin lesions diagnostics via integrated deep convolutional networks for segmentation and classification. Computer methods and programs in biomedicine 190, 105351. 
Araújo, R.L., Ricardo de Andrade, L.R., Rodrigues, J.J., e Silva, R.R., 2021. Automatic segmentation of melanoma skin cancer using deep learning, in: 2020 IEEE International Conference on E-health Networking, Application \& Services (HEALTHCOM), IEEE. pp. 1-6.

Arora, R., Raman, B., Nayyar, K., Awasthi, R., 2021. Automated skin lesion segmentation using attention-based deep convolutional neural network. Biomedical Signal Processing and Control 65, 102358.

Caselles, V., Kimmel, R., Sapiro, G., 1997. Geodesic active contours. International Journal of Computer Vision 22, 61-79. doi 10.1023/A : 1007979827043

Celebi, M., Wen, Q., Iyatomi, H., Shimizu, K., Zhou, H., Schaefer, G., 2015. A state-of-the-art survey on lesion border detection in dermoscopy images, in: Dermoscopy Image Analysis. CRC Press, pp. 97-129. URL: https: //doi.org/10.1201\%2Fb19107-5 doi 10.1201/b19107-5

Chan, S., Reddy, V., Myers, B., Thibodeaux, Q., Brownstone, N., Liao, W., 2020. Machine learning in dermatology: Current applications, opportunities, and limitations. Dermatology and Therapy , 6869-6888.

Chu, A., Sehgal, C.M., Greenleaf, J.F., 1990. Use of gray value distribution of run lengths for texture analysis. Pattern Recognition Letters 11, 415-419.

Codella, N.C.F., Gutman, D., Celebi, M.E., Helba, B., Marchetti, M.A., Dusza, S.W., Kalloo, A., Liopyris, K., Mishra, N., Kittler, H., Halpern, A., 2018. Skin lesion analysis toward melanoma detection: A challenge at the 2017 international symposium on biomedical imaging (isbi), hosted by the international skin imaging collaboration (isic), in: 2018 IEEE 15th International Symposium on Biomedical Imaging (ISBI 2018), pp. 168-172.

Codella, N.C.F., Gutman, D., Celebi, M.E., Helba, B., Marchetti, M.A., Dusza, S.W., Kalloo, A., Liopyris, K., Mishra, N.K., Kittler, H., Halpern, A., 2017. Skin lesion analysis toward melanoma detection: A challenge at the 2017 international symposium on biomedical imaging (isbi), hosted by the international skin imaging collaboration (ISIC). IEEE arXiv:1710.05006

Dasarathyand, B.R., Holder, E.B., 1991. Image characterizations based on joint gray-level run-length distributions. Pattern Recognition Letters 12, 497-502.

Diepgen, T.L., Yihune, G., 2012. Dermatology information system - dermis. http://dermis.net/ Retrieved September 12, 2016.

Filali, I., Belkadi, M., 2019. Multi-scale contrast based skin lesion segmentation in digital images. Optik 185, 794 - 811. URL: http://www. sciencedirect.com/science/article/pii/S0030402619304917. doi https://doi.org/10.1016/j.ijleo.2019.04.022

Fulgencio Navarro, M.E.V., Bescós, J., 2019. Accurate segmentation and registration of skin lesion images to evaluate lesion change. IEEE Journal of Biomedical and Health Inf 23, $501-508$.

Galloway, M.M., 1975. Texture analysis using gray level run lengths. Computer graphics and image processing 4, 172-179.

Garcia-Arroyo, J.L., Garcia-Zapirain, B., 2019. Segmentation of skin lesions in dermoscopy images using fuzzy classification of pixels and histogram thresholding. Computer Methods and Programs in Biomedicine 168, 11 19. doi https://doi.org/10.1016/j.cmpb.2018.11.001

Goyal, M., Oakley, A., Bansal, P., Dancey, D., Yap, M.H., 2020. Skin lesion segmentation in dermoscopic images with ensemble deep learning methods. IEEE Access 8, 4171-4181.

Gutman, D., Codella, N.C.F., Celebi, M.E., Helba, B., Marchetti, M.A., Mishra, N.K., Halpern, A., 2016. Skin lesion analysis toward melanoma detection: A challenge at the international symposium on biomedical imaging (ISBI) 2016, hosted by the international skin imaging collaboration (ISIC). arXiv URL: arXiv: 1605.01397 arXiv:1605.01397

Halder, A., Chatterjee, S., Dey, D., Kole, S., Munshi, S., 2020. An adaptive morphology based segmentation technique for lung nodule detection in thoracic ct image. Computer Methods and Programs in Biomedicine 197, 105720.

Haralick, R.M., Shanmugam, K., Dinstein, I., 1973. Textural features for image classification. IEEE Transactions on Systems, Man, and Cybernetics SMC3, 610-621.

He, D., Chen, Y., 2017. Anomaly detection based on super-pixels time context feature, in: 2017 29th Chinese Control And Decision Conference (CCDC), IEEE. pp. 1329-1334.

Hemalatha, R., Thamizhvani, T., Dhivya, A.J.A., Joseph, J.E., Babu, B., Chandrasekaran, R., 2018. Active contour based segmentation techniques for medical image analysis, in: Koprowski, R. (Ed.), Medical and Biological Image Analysis. IntechOpen, Rijeka. chapter 2, pp. 17-34. doi 10.5772/ intechopen.74576

Hu, K., Liu, S., Zhang, Y., Cao, C., Xiao, F., Huang, W., Gao, X., 2020. Automatic segmentation of dermoscopy images using saliency combined with adaptive thresholding based on wavelet transform. Multimedia Tools and Applications 79, 14625-14642.

Isaaks, E.H., Srivastava, R.M., 1989. An introduction to Applied Geostatistics. Oxford University Press.

Khalid, S., Jamil, U., Saleem, K., Akram, M.U., Manzoor, W., Ahmed, W., Sohail, A., 2016. Segmentation of skin lesion using cohen-daubechies-feauveau biorthogonal wavelet. SpringerPlus 5, 1-17.

Lacy, K., Alwan, W., 2013. Skin cancer. Medicine 41, 402-405.

Lee, T., Ng, V., Gallagher, R., Coldman, A., McLean, D., 1997. Dullrazorß: A software approach to hair removal from images. Computers in biology and medicine 27, 533-543.

Lei, B., Xia, Z., Jiang, F., Jiang, X., Ge, Z., Xu, Y., Qin, J., Chen, S., Wang, T., Wang, S., 2020. Skin lesion segmentation via generative adversarial networks with dual discriminators. Medical Image Analysis 64, 101716.

Li, X., Chen, H., Qi, X., Dou, Q., Fu, C., Heng, P., 2018. H-denseunet: Hybrid densely connected unet for liver and tumor segmentation from ct volumes. IEEE Transactions on Medical Imaging 37, 2663-2674. doi 10.1109/TMI. 2018.2845918

Li, X., Yu, L., Chen, H., Fu, C., Heng, P., 2018. Semi-supervised skin lesion segmentation via transformation consistent self-ensembling model. arXiv: 1808.03887 arXiv: 1808.03887

Li, Y., Shen, L., 2018. Skin lesion analysis towards melanoma detection using deep learning network. Sensors 18, 556. URL: http://dx.doi.org/10. $3390 / \mathrm{s} 18020556$ doi $10.3390 / \mathrm{s} 18020556$

López Sarachaga, C., Lage Medina, S., Morales González, M.C., Dolores, B.L.M., Asumendi Mallea, A., Garrote Contreras, E., Conde Portilla, O.M., et al., 2019. Automatic pigmented lesion segmentation through a dermoscopy-guided oct approach for early diagnosis, SPIE Society of Photo-Optical Instrumentation Engineers.

Ma, Z., Tavares, J.M.R.S., 2016. A novel approach to segment skin lesions in dermoscopic images based on a deformable model. Journal of Biomedical and Health Informatics 20, 615-623.

MATLAB, 2010. version 7.10.0 (R2010a). The MathWorks Inc., Natick, Massachusetts.

Mendonça, T., Ferreira, P.M., Marques, J.S., Marcal, A.R., Rozeira, J., 2013. $\mathrm{Ph} 2$-a dermoscopic image database for research and benchmarking, in: Engineering in Medicine and Biology Society (EMBC), 2013 35th Annual International Conference of the IEEE, IEEE. pp. 5437-5440.

Moura, N., Veras, R., Aires, K., Machado, V., Silva, R., Araújo, F., Claro, M., 2019. Abcd rule and pre-trained cnns for melanoma diagnosis. Multimedia Tools Appl. 78, 6869-6888. URL: https://doi.org/10.1007/ s11042-018-6404-8 doi 10.1007/s11042-018-6404-8

Moura, N., Veras, R., Aires, K., Machado, V., Silva, R., Araújo, F., Claro, M., 2018. Combining abcd rule, texture features and transfer learning in automatic diagnosis of melanoma, in: 2018 IEEE Symposium on Computers and Communications (ISCC), pp. 00508-00513. doi 10.1109/ISCC. 2018.8538525

Nan, B.F., Mu, Z.C., 2014. Slico-based superpixel segmentation method with texture fusion. Chinese Journal of Scientific Instrument 35, 527-534.

Nida, N., Irtaza, A., Javed, A., Yousaf, M.H., Mahmood, M., 2019. Melanoma lesion detection and segmentation using deep region based convolutional neural network and fuzzy c-means clustering. International Journal of Medical Informatics 124. doi $10.1016 / j$. ijmedinf .2019.01.005

Ojala, T., Pietikäinen, M., Harwood, D., 1996. A comparative study of texture measures with classification based on featured distributions. Pattern recognition 29, 51-59.

Oliveira, R.B., Filho, M.E., Ma, Z., Papa, J.P., Pereira, A.S., Tavares, J.M.R., 2016. Computational methods for the image segmentation of pigmented skin lesions: A review. Computer Methods and Programs in Biomedicine 131, $127-141$.

Organization, W.H., 2019. Skin cancers. https://www.who.int/uv/faq/ skincancer/en/index1.html

Pennisi, A., Bloisi, D.D., Nardi, D., Giampetruzzi, A.R., Mondinoc, C., Facchiano, A., 2016. Skin lesion image segmentation using delaunay triangulation for melanoma detection. Computerized Medical Imaging and Graphics 52, 89-103.

Pereira, P.M., Fonseca-Pinto, R., Paiva, R.P., Assuncao, P.A., Tavora, L.M., Thomaz, L.A., Faria, S.M., 2020. Dermoscopic skin lesion image segmentation based on local binary pattern clustering: comparative study. Biomedical Signal Processing and Control 59, 101924.

Qamar, S., Ahmad, P., Shen, L., 2021. Dense encoder-decoder-based architecture for skin lesion segmentation. Cognitive Computation 13, 583-594. 
Quinlan, J.R., 1986. Induction of decision trees. Machine learning 1, 81-106.

Raileanu, L.E., Stoffel, K., 2004. Theoretical comparison between the gini index and information gain criteria. Annals of Mathematics and Artificial Intelligence 41, 77 - 93. doi https://doi.org/10.1023/B:AMAI. $0000018580.96245 . c 6$

Ross, T.J., 2009. Fuzzy logic with engineering applications. John Wiley \& Sons.

Rother, C., Kolmogorov, V., Blake, A., 2004. "grabcut": Interactive foreground extraction using iterated graph cuts. ACM Trans. Graph. 23, 309-314. URL: https://doi.org/10.1145/1015706.1015720 doi 10.1145/ 1015706.1015720

Santos, L., Veras, R., Aires, K., Britto, L., Machado, V., 2018. Medical image segmentation using seeded fuzzy c-means: A semi-supervised clustering algorithm., in: International Joint Conference on Neural Networks, IEEE. pp. $1-8$.

Scharcanski, J., Celebi, M.E., 2013. Computer vision techniques for the diagnosis of skin cancer. Springer.

Siegel, R.L., Miller, K.D., Fuchs, H.E., Jemal, A., 2021. Cancer statistics, 2021. CA: A Cancer Journal for Clinicians 71, 7-33. URL: https://acsjournals.onlinelibrary.wiley.com/doi/abs/ 10.3322/caac.21654 doi/https://doi.org/10.3322/caac.21654

Silva, A.C., Carvalho, P.C.P., Gattass, M., 2004. Analysis of spatial variability using geostatistical functions for diagnosis of lung nodule in computerized tomography images. Pattern Analysis and Applications 7, 227-234.

Sousa, J., Paiva, A., Almeida, J., Silva, A., Junior, G., Gattass, M., 2017. Texture based on geostatistic for glaucoma diagnosis from fundus eye image. Multimedia Tools and Applications 76. doi 10.1007/ s11042-017-4608-y

Sultana, N.N., Puhan, N.B., 2018. Recent deep learning methods for melanoma detection: A review, in: Ghosh, D., Giri, D., Mohapatra, R.N., Savas, E., Sakurai, K., Singh, L.P. (Eds.), Mathematics and Computing, Springer Singapore, Singapore. pp. 118-132.

Tamura, H., Mori, S., Yamawaki, T., 1978. Textural features corresponding to visual perception. IEEE Transactions on Systems, Man, and Cybernetics 8, 460-473.

Tong, X., Wei, J., Sun, B., Su, S., Zuo, Z., Wu, P., 2021. Ascu-net: Attention gate, spatial and channel attention u-net for skin lesion segmentation. Diagnostics 11, 501.

Vasconcelos, F.F.X., Medeiros, A.G., Peixoto, S.A., Filho, P.P.R., 2019. Automatic skin lesions segmentation based on a new morphological approach via geodesic active contour. Cognitive Systems Research 55, 44 - 59. doi https://doi.org/10.1016/j. cogsys . 2018.12.008

Wu, H., Pan, J., Li, Z., Wen, Z., Qin, J., 2020. Automated skin lesion segmentation via an adaptive dual attention module. IEEE Transactions on Medical Imaging 40, 357-370.

Xie, Y., Zhang, J., Xia, Y., Shen, C., 2019. Semi- and weakly supervised directional bootstrapping model for automated skin lesion segmentation. CoRR abs/1903.03313. URL: http://arxiv.org/abs/1903.03313 arXiv: 1903.03313

Xie, Y., Zhang, J., Xia, Y., Shen, C., 2020. A mutual bootstrapping model for automated skin lesion segmentation and classification. IEEE Transactions on Medical Imaging , 1-1.

Ünver, H.M., Ayan, E., 2019. Skin lesion segmentation in dermoscopic images with combination of yolo and grabcut algorithm. Diagnostics 9, 72. URL: http://dx.doi.org/10.3390/diagnostics9030072 doi $10.3390 /$ diagnostics 9030072 